MODELING, IDENTIFICATION AND CONTROL, 1990, VOL. 11, NO. 2, 89-96

doi:10.4173/mic.1990.2.2

\title{
A multivariable robust adaptive controller using reduced-order model
}

\begin{abstract}
WEI WANG $\dagger$
Keywords: Adaptive control, multivariable systems, stability, robustness.

In this paper a multivariable robust adaptive controller is presented for a plant with bounded disturbances and unmodeled dynamics due to plant-model order mismatches. The robust stability of the closed-loop system is achieved by using the normalization technique and the least squares parameter estimation scheme with dead zones. The weighting polynomial matrices are incorporated into the control law, so that the open-loop unstable or/and nonminimum phase plants can be handled.
\end{abstract}

\section{Introduction}

Most adaptive control systems are designed with the assumption that the plant order is exactly known. However this is a critical restriction in practical application of adaptive control since, in general, the plant order cannot be known exactly. It is then natural to ask how an adaptive control system behaves when the plant is not perfectly described by a given model. In addition, there are usually unmeasured bounded disturbances, such as step load disturbances acting on plants. It was shown that such unmodeled dynamics, however small, may cause instability in an adaptive control system (Rohrs et al. 1982). Therefore considerable research has been attracted to this field (Ortega and Yu 1987). Generally, the normalization and parameter estimation with a dead zone are used in the design of a robust adaptive controller. Cluett $e t$ al. $(1987,1988)$ suggested the robust adaptive controllers for single-input single-output systems with a plant-model order mismatch and bounded disturbances by using a projection parameter estimation or a least-squares parameter estimation.

The results reported in this paper differ from the related work in Cluett $e$ al. (1987, 1988) as follows. (1) A robust adaptive controller for multi-input multi-output systems with plant-model order mismatches and bounded disturbances is suggested. (2) Although an augmented plant was used in Cluett $e$ t al. (1987) for incorporating the weighting polynomials $P, Q$ and $R$ into the predictive control law in order to handle some nonminimum phase plants, a weakness is that the original plant must be openloop stable. This limits the use of the suggested robust controller. Here the concept of the generalized minimum variance with the weighting polynomial matrices $P, Q$ and $R$ (Koivo, 1980) is considered in the design of the robust adaptive controller. The advantage is that the open-loop unstable or/and nonminimum phase systems can be handled even in the existence of plant-model order mismatch and bounded disturbances.

Received 15 September 1990.

† Division of Engineering Cybernetics, The Norwegian Institute of Technology, N-7034 Trondheim, Norway, on leave from Department of Automatic Control, Northeast University of Technology, Shenyang, 110006, P.R. China.

This work was supported by the Royal Norwegian Council for Scientific and Industrial Research. 


\section{A MIMO adaptive control scheme}

\subsection{Plant description}

Consider a MIMO linear discrete-time plant, with constant but unknown parameters, represented by the form

$$
A\left(q^{-1}\right) y(t)=B\left(q^{-1}\right) u(t-k)+\xi(t)
$$

where $y(t) \in \mathbf{R}^{m}, u(t) \in \mathbf{R}^{m}$ and $\xi(t) \in \mathbf{R}^{m}$ are plant output, input and bounded disturbance vectors respectively, $A\left(q^{-1}\right)=\operatorname{diag}\left(A_{i}\left(q^{-1}\right)\right)$ and $B\left(q^{-1}\right)$ is a polynomial matrix, where $A_{i}\left(q^{-1}\right)(1 \leqslant i \leqslant m), B_{i j}\left(q^{-1}\right)(1 \leqslant i \leqslant m, 1 \leqslant j \leqslant m)$ are polynomials in the backward-shift operator $q^{-1}$ and $A_{i}(0) \neq 0$. $k$ is the time delay of the plant. Using the polynomial matrix equation

$$
P\left(q^{-1}\right)=F\left(q^{-1}\right) A\left(q^{-1}\right)+q^{-k} G\left(q^{-1}\right)
$$

where $P\left(q^{-1}\right)=\operatorname{diag}\left(P_{i}\left(q^{-1}\right)\right), F\left(q^{-1}\right)=\operatorname{diag}\left(F_{i}\left(q^{-1}\right)\right)$. $G\left(q^{-1}\right)=\operatorname{diag}\left(G_{i}\left(q^{-1}\right)\right)$ and the order of $F\left(q^{-1}\right)$ and $G\left(q^{-1}\right)$ are $k-1$ and $n_{G}, n_{G}=\max \left(n_{P}-k, n_{A}-1\right)$. Using (2), equation (1) is written as

$$
P\left(q^{-1}\right) y(t+k)=\alpha\left(q^{-1}\right) y(t)+\beta\left(q^{-1}\right) u(t)+\delta(t+k)
$$

where $\alpha\left(q^{-1}\right)=G\left(q^{-1}\right), \beta\left(q^{-1}\right)=F\left(q^{-1}\right) B\left(q^{-1}\right), \delta(t+k)=F\left(q^{-1}\right) \xi(t+k)$. It is clear that $\delta(t)$ still is a bounded disturbance vector. Equation (3) can be written as

$$
P_{i}\left(q^{-1}\right) y_{i}(t+k)=X_{i}(t)^{\mathrm{T}} \Omega_{i}+\delta_{i}(t+k), \quad i=1, \ldots, m
$$

where

$$
\begin{aligned}
\Omega_{i}^{\mathrm{T}} & =\left[\alpha_{0}^{i}, \ldots, \alpha_{r}^{i} \quad \beta_{0}^{i 1}, \ldots, \beta_{0}^{i m}, \ldots ; \beta_{s}^{i 1}, \ldots, \beta_{s}^{i m}\right] \\
X_{i}(t)^{\mathrm{T}} & =\left[y_{i}(t), \ldots, y_{i}(t-r), u(t)^{\mathrm{T}}, \ldots, u(t-s)^{\mathrm{T}}\right]
\end{aligned}
$$

and $r=n_{G}, s=n_{G}+k-1$.

Most actual plants are high order and in general, the model order is often chosen to be less than the actual plant order for simplifying the design of the controller and implementing the algorithm easily, even in non-adaptive cases. Suppose we choose $r_{1} \leqslant r$ and $s_{1} \leqslant s$ as the model order, then (4) is rewritten as

$$
P_{i}\left(q^{-1}\right) y_{i}(t)=X_{i}(t-k)^{\mathrm{T}} \Omega_{i}+\delta_{i}(t)=x_{i}(t-k)^{\mathrm{T}} \theta_{i}+z_{i}(t-k)^{\mathrm{T}} \rho_{i}+\delta_{i}(t), i=1, \ldots, m
$$
where

$$
\begin{gathered}
\Omega_{i}^{\mathrm{T}}=\left[\theta_{i}^{\mathrm{T}} ; \rho_{i}^{\mathrm{T}}\right]=\left[\alpha_{0}^{i}, \ldots, \alpha_{r_{1}}^{i} ; \beta_{0}^{i 1}, \ldots, \beta_{0}^{i m} ; \ldots ; \beta_{s_{1}}^{i 1}, \ldots, \beta_{s_{1}}^{i m} ; \alpha_{r_{1}+1}^{i}, \ldots, \alpha_{r}^{i} ;\right. \\
\left.\beta_{s_{1}+1}^{i 1}, \ldots, \beta_{s 1+1}^{i m} ; \ldots ; \beta_{s}^{i 1}, \ldots, \beta_{s}^{i m}\right] \\
X_{i}(t)^{\mathrm{T}}=\left[x_{i}(t)^{\mathrm{T}} ; z_{i}(t)^{\mathrm{T}}\right]=\left[y_{i}(t), \ldots, y_{i}\left(t-r_{1}\right) ;\right. \\
\left.u(t)^{\mathrm{T}}, \ldots, u\left(t-s_{1}\right)^{\mathrm{T}} ; y_{i}\left(t-r_{1}-1\right), \ldots, y_{i}(t-r) ; u\left(t-s_{1}-1\right)^{\mathrm{T}}, \ldots, u(t-s)^{\mathrm{T}}\right]
\end{gathered}
$$

\subsection{Normalized model}

If the unmodeled dynamics $z_{i}(t-k)^{\mathrm{T}} \rho_{i}$ in (5) are bounded they can be absorbed in the bounded disturbances $\delta_{i}(t)$. It is evident that $z_{i}(t-k)^{\mathrm{T}} \rho_{i}$ may be unbounded since $z_{i}(t-k)$ involves $y_{i}($.$) and u($.$) . In order to guarantee the boundedness of z_{i}(t-k)^{\mathrm{T}} \rho_{i}$, similar to Cluett et al. $(1987,1988)$, the normalization technique is used here. The normalized variable is defined as follows.

$$
*_{i}^{n}(t)=*_{i}(t) / n_{i}(t)
$$




$$
n_{i}(t)=\max \left[\max _{1 \leqslant j \leqslant h_{2}}\left|\left[\chi_{i}(t-k)\right]_{j}\right|, C\right]
$$

where

$$
\chi_{i}(t)^{\mathrm{T}}=\left[y_{i}(t), \ldots, y_{i}\left(t-r_{2}\right), u(t)^{\mathrm{T}}, \ldots, u\left(t-s_{2}\right)^{\mathrm{T}}\right]
$$

and $h_{2}=r_{2}+s_{2}$ with $r \leqslant r_{2}$ and $s \leqslant s_{2} .\left[\chi_{i}(t-k)\right]_{j}$ is the jth element of $\chi_{i}(t-k)$ and $C$, used to prevent a division by zero, is any positive constant. Equation (5) is divided by $n_{i}(t)$ then we have

$$
P_{i}\left(q^{-1}\right) y_{i}^{n}(t)=x_{i}^{n}(t-k)^{\mathrm{T}} \theta_{i}+d_{i}^{n}(t), i=1, \ldots, m
$$

where

$$
d_{i}^{n}(t)=z_{i}^{n}(t-k)^{\mathbf{T}} \rho_{i}+\delta_{i}^{n}(t)
$$

The normalization used here has the advantage of guaranteeing the boundedness of $x_{i}^{n}(t-k)$ and $z_{i}^{n}(t-k)$. Noting that $\left\|\rho_{i}\right\|$ is bounded and $n_{i}(t) \geqslant C$, it is clear that $d_{i}^{n}(t)$ is bounded. Suppose $M_{i}$ to be an upper bound of $\left|d_{i}^{n}(t)\right|$, i.e.

$$
\sup \left|d_{i}^{n}(t)\right| \leqslant M_{i}, \forall t \geqslant 0, i=1, \ldots, m
$$

\subsection{Robust adaptive controller}

When parameters of the plant (1) are unknown, we use the estimate $\hat{\theta}_{i}(t)$ instead of $\theta_{i}$. Define $\varepsilon_{i}(t)$ as an estimation error

$$
\varepsilon_{i}(t)=P_{i}\left(q^{-1}\right) y_{i}(t)-x_{i}(t-k)^{\mathrm{T}} \hat{\theta}_{i}(t-1), i=1, \ldots, m
$$

Then the normalized $\varepsilon_{i}(t)$ is

$$
\varepsilon_{i}^{n}(t)=P_{i}\left(q^{-1}\right) y_{i}^{n}(t)-x_{i}^{n}(t-k)^{\mathrm{T}} \hat{\theta}_{i}(t-1)
$$

In order to handle the bounded variable $d_{i}^{n}(t)$ the recursive least-squares parameter estimation scheme with dead zones given in Gu and Wang (1988) is used for the normalized reduced-order model (8). for $i=1, \ldots, m$

$$
\begin{gathered}
\hat{i}_{i}(t)=\hat{\theta}_{i}(t-1)+\frac{\lambda_{i}(t) P_{i}(t-2) x_{i}^{n}(t-k) \varepsilon_{i}^{n}(t)}{1+x_{i}^{n}(t-k)^{\mathrm{T}} P_{i}(t-2) x_{i}^{n}(t-k)} \\
P_{i}(t-1)=P_{i}(t-2)-\frac{\lambda_{i}(t) P_{i}(t-2) x_{i}^{n}(t-k) x_{i}^{n}(t-k)^{\mathrm{T}} P_{i}(t-2)}{1+x_{i}^{n}(t-k)^{\mathrm{T}} P_{i}(t-2) x_{i}^{n}(t-k)} \\
\lambda_{i}(t)= \begin{cases}0 & \text { if }\left|\varepsilon_{i}^{n}(t)\right|<2 M_{i} \\
\gamma & \text { otherwise } \gamma \in(\tau, 3(1-\tau) / 4)\end{cases}
\end{gathered}
$$

where $0<\tau<3 / 7$.

The following adaptive control law is adopted.

$$
x(t)^{\mathrm{T}} \hat{\theta}(t)-R\left(q^{-1}\right) w(t+k)+Q\left(q^{-1}\right) u(t)=0
$$

where $R\left(q^{-1}\right)$ and $Q\left(q^{-1}\right)$ are polynomial matrices in $q^{-1}, w(t)$ is a bounded reference vector and

$$
\begin{aligned}
& x(t)=\operatorname{diag}\left[x_{1}(t), \ldots, x_{m}(t)\right] \\
& \hat{\theta}(t)=\operatorname{diag}\left[\hat{\theta}_{1}(t), \ldots, \hat{\theta}_{m}(t)\right]
\end{aligned}
$$


Remark 1. An advantage of the recursive least-squares parameter estimation scheme with dead zones used here is to ensure that the coefficient matrix of $u(t)$ in (15) is nonsingular for all $t$ by the choice of $\gamma$ in (14) (Gu and Wang, 1988).

Remark 2. We know from Koivo (1980) that the adaptive control law in (15) is meaningful because when the parameters are known and $\xi(t)$ is the zero-mean white noise vector, the generalized minimum variance control of the plant (1) is given by

$$
X(t)^{\mathrm{T}} \Omega-R\left(q^{-1}\right) w(t+k)+Q\left(q^{-1}\right) u(t)=0
$$

which implies minimization of the cost function

$$
J=E\left\{\left[P\left(q^{-1}\right) y(t+k)-R\left(q^{-1}\right) w(t+k)+Q\left(q^{-1}\right) u(t)\right]^{2}\right\}
$$

The cost function with the weighting polynomials $P\left(q^{-1}\right), R\left(q^{-1}\right)$ and $Q\left(q^{-1}\right)$ was first suggested by Clarke and Gawthrop $(1975,1979)$ for a self-tuning controller. The adaptive controller presented here, however, is not of minimum variance because of the presence of unmodeled dynamics and bounded disturbances.

\section{Stability analysis}

\subsection{Basic assumptions}

The basic assumptions necessary for the stability analysis in the paper are

A1: The time delay $k$ is known.

A2. The upper bounds of the order of $A\left(q^{-1}\right)$ and $B\left(q^{-1}\right)$ are known.

A . The upper bound of $\left|d_{i}(t)\right|$, i.e. $\mathbf{M}_{i},(i=1, \ldots, m)$, are known.

A4: The off-line choices of $P\left(q^{-1}\right)$ and $Q\left(q^{-1}\right)$ are such that the polynomial matrix

$$
T\left(q^{-1}\right)=P\left(q^{-1}\right) B\left(q^{-1}\right)+Q\left(q^{-1}\right) A\left(q^{-1}\right)
$$

is stable, i.e. $\operatorname{det} T(z)=0$ for $|z|>0$.

Note the assumption A2 does not require knowledge of the plant order, but only an upper bound of its order. The assumption A2 is used here to decide $r_{2}$ and $s_{2}$, which is the dimension of $\chi_{i}(t)$ in the normalization factor $n_{i}(t)$.

\subsection{Convergence properties of the normalized model}

Some convergence properties of the normalized model (8) are given below.

Lemma 1. The estimation scheme (12)-(14) has the following properties.

(i) $v_{i}(t)-v_{i}(t-1) \leqslant 0$,

(ii) $\lim _{t \rightarrow \infty} \lambda_{i}(t) \varepsilon_{i}^{n}(t)^{2}=0$.

where $v_{i}(t)=\tilde{\theta}_{i}(t)^{\mathrm{T}} P_{i}(t-1)^{-1} \tilde{\theta}_{i}(t), \tilde{\theta}_{i}(t)=\theta_{i}(t)-\theta_{i}, i=1, \ldots, m$.

Proof. See Gu and Wang (1988).

Define the generalized error vector $e(t)$

$$
e(t)=P y(t)-R w(t)+Q u(t-k)
$$

Substituting (10) and (15) into (16) yields

$$
e(t)=\varepsilon(t)+x(t-k)^{\mathrm{T}}[\hat{\theta}(t-1)-\theta(t-k)]
$$


Then the normalized form of $e_{i}(t)$ is

$$
e_{i}^{n}(t)=\varepsilon_{i}^{n}(t)+x_{i}^{n}(t-k)^{\mathrm{T}}\left[\hat{\theta}_{i}(t-1)-\hat{\theta}_{i}(t-k)\right]
$$

Lemma 2. If the estimation scheme (12)-(14) is applied to the normalized system (8), there exists $T>0$ and for $t \geqslant T, i=1, \ldots, m$,

(i) $\left\|\hat{\theta}_{i}(t-k)-\hat{\theta}_{i}(t-1)\right\|=0$,

(ii) $\left|e_{i}(t)\right|<2 M_{i} n_{i}(t)$.

Proof. Define $H_{i}=\left\{t \mid \lambda_{i}(t) \neq 0, t \in N^{+}\right\}$for every $i(1 \leqslant i \leqslant m) . N^{+}$represents a set of natural numbers. We can prove that $H_{i}$ must be a finite set. Otherwise there is at least one $i_{0}\left(1 \leqslant i_{0} \leqslant m\right)$ and its corresponding $H_{i 0}$ is infinite. Then $H_{i_{0}}$ can be represented by a series of $\left\{t_{n}\right\}$ and $t_{n} \rightarrow \infty(n \rightarrow \infty)$. From (14) we have

$$
\lambda_{i_{0}}\left(t_{n}\right) \geqslant \tau>0
$$

Then from Lemma 1 (ii) it follows readily that

$$
\lim _{n \rightarrow \infty} \varepsilon_{i_{0}}^{n}\left(t_{n}\right)=0
$$

When $n$ is large enough, it must be

$$
\left|\varepsilon_{i_{0}}^{n}\left(t_{n}\right)\right|<2 M_{i_{0}}
$$

From (14) it follows that $\lambda_{i_{0}}\left(t_{n}\right)=0$. This contradicts (21). Then for every $i(1 \leqslant i \leqslant m) H_{i}$ is finite. Thus there exists $t_{i} \geqslant 0$ and when $t \geqslant t_{i}, \lambda_{i}(t)=0$. Let

$$
T=\max _{1 \leqslant i \leqslant m}\left\{t_{i}+k\right\}
$$

and from (12) and (14) it yields

$$
\hat{\theta}_{i}(t-k)=\hat{\theta}_{i}(t-1), t \geqslant T
$$

which means the result (i). From (14), (18), (19) and the boundedness of $x_{i}^{n}(t-k)$ we have result (ii).

\subsection{Stability results}

Introducing polynomial matrices $\bar{A}\left(q^{-1}\right), \bar{B}\left(q^{-1}\right), \widetilde{B}\left(q^{-1}\right)$ and $\tilde{Q}\left(q^{-1}\right)$ such that

$$
\begin{aligned}
\left.A\left(q^{-1}\right) \bar{B} q^{-1}\right) & =B\left(q^{-1}\right) \bar{A}\left(q^{-1}\right) \\
\tilde{B}\left(q^{-1}\right) Q\left(q^{-1}\right) & =\tilde{Q}\left(q^{-1}\right) B\left(q^{-1}\right)
\end{aligned}
$$

where $\operatorname{det} \bar{A}=\operatorname{det} A, \bar{A}(0)=A(0), \operatorname{det} \widetilde{B}=\operatorname{det} B, \widetilde{B}(0)=B(0)$. We know that $\bar{A}\left(q^{-1}\right)$, $\bar{B}\left(q^{-1}\right), \widetilde{B}\left(q^{-1}\right)$ and $\tilde{Q}\left(q^{-1}\right)$ always exist (Wolovich, 1974).

Multiplying (16) on the left by $A$ and using (1) we have

$$
(P B+A Q) u(t-k)=A e(t)+\Delta_{1}(t)
$$

where

$$
\Delta_{1}(t)=A R w(t)-P \xi(t)
$$

Multiplying (16) on the left by $\widetilde{B}$ and using (1) and (23) we have

$$
(\tilde{B} P+\tilde{Q} A) y(t)=\tilde{B} e(t)+\Delta_{2}(t)
$$


where

$$
\Delta_{2}(t)=\tilde{B} R w(t)+\tilde{Q} \xi(t)
$$

From (22) and (23) we have

$$
\begin{aligned}
\operatorname{det}(\tilde{B P}+\widetilde{Q A}) & =\operatorname{det}\left[\widetilde{B}\left(P+Q B^{-1} A\right)\right]=\operatorname{det}\left[\widetilde{B}(P \bar{B}+Q \bar{A}) \bar{B}^{-1}\right] \\
& =\operatorname{det}\left[\left(P A^{-1} B+Q\right) \bar{A}\right]=\operatorname{det}\left[A^{-1}(P B+A Q) \bar{A}\right] \\
& =\operatorname{det}(P B+A Q)
\end{aligned}
$$

Lemma 3. Under the assumption A1-A4 there exist positive constants $C_{1}$ and $C_{2}$ such that

$$
C_{1}\|\chi(t-k)\| \leqslant C_{2}+\max _{0 \leqslant \tau \leqslant N, 1 \leqslant j \leqslant m}\left|e_{j}(\tau)\right|, 0 \leqslant t \leqslant N
$$

where

$$
\chi(t)^{\mathrm{T}}=\left[\chi_{1}(t)^{T}, \ldots, \chi_{m}(t)^{\mathrm{T}}\right]
$$

Proof. Since $\Delta_{1}(t)$ and $\Delta_{2}(t)$ are bounded, according to assumption A4 and Lemma 3.2 (Goodwin $e t$ al. 1980), (24)-(26) imply that for all $t$ and $i=1, \ldots, m$

$$
\begin{array}{r}
\left|y_{i}(t)\right| \leqslant K_{1}+K_{2} \max _{0 \leqslant \tau \leqslant N, 1 \leqslant j \leqslant m}\left|e_{j}(\tau)\right|, 0 \leqslant t \leqslant N \\
\left|u_{i}(t-k)\right| \leqslant K_{3}+K_{4} \max _{0 \leqslant \tau \leqslant N, 1 \leqslant j \leqslant m}\left|e_{j}(\tau)\right|, 0 \leqslant t \leqslant N
\end{array}
$$

Then (28) and (29) imply that

$$
\|\chi(t-k)\| \leqslant K_{5}+K_{6} \max _{0 \leqslant \tau \leqslant N, 1 \leqslant j \leqslant m}\left|e_{j}(\tau)\right|, 0 \leqslant t \leqslant N
$$

which means

$$
C_{1}\|\chi(t-k)\| \leqslant C_{2}+\max _{0 \leqslant \tau \leqslant N, 1 \leqslant j \leqslant m}\left|e_{j}(\tau)\right| \quad 0 \leqslant t \leqslant N
$$

where $C_{1}=1 / K_{6}$ and $C_{2}=K_{5} / K_{6}$.

It is obvious that the linear boundedness condition (27) depends on the location of the roots of the polynomial det $T\left(q^{-1}\right)$. For any given $A\left(q^{-1}\right)$ and $B\left(q^{-1}\right)$ it is possible to select the polynomial matrices $P\left(q^{-1}\right)$ and $Q\left(q^{-1}\right)$ to satisfy this condition.

The stability results of the adaptive control system in the presence of bounded disturbances and unmodeled dynamics are contained in the following theorem.

Theorem 1. Under the basic assumptions A1-A4 and if

$$
C_{1}>2 M, \text { where } M=\max _{1 \leqslant i \leqslant m} M_{i}
$$

then the adaptive control system formed by the plant (1), the estimation scheme (12) - (14) and control law (15) have the following properties

(i) $\{\|y(t)\|\}$ and $\{\|u(t)\|\}$ are bounded.

(ii) $\left|e_{i}(t)\right|<2 M_{i} n_{i}(t), t \geqslant T, i=1, \ldots, m$. 
Proof. Lemma 2 (ii) and (30) yield

$$
\begin{aligned}
C_{1}>2 M & >\max _{1 \leqslant i \leqslant m}\left\{\left|e_{i}(t)\right| / n_{i}(t)\right\} \\
& \geqslant \max _{1 \leqslant i \leqslant m}\left\{\left|e_{i}(t)\right| / \max \left\{\left\|\chi_{i}(t-k)\right\|, C\right\}\right\} \\
& \geqslant \max _{1 \leqslant i \leqslant m}\left|e_{i}(t)\right| / \max \{\|\chi(t-k)\|, C\}
\end{aligned}
$$

Assume the sequence $\{\|\chi(t)\|\}$ to be unbounded. From lemma 3 there exists a subsequence $\left\{t_{n}\right\}$ such that

$$
C_{1}\left\|\chi\left(t_{n}-k\right)\right\| \leqslant C_{2}+\max _{1 \leqslant i \leqslant m}\left|e_{i}\left(t_{n}\right)\right|
$$

Dividing both sides by $\left\|\chi\left(t_{n}-k\right)\right\|$ we obtain

$$
C_{1} \leqslant C_{2} /\left\|\chi\left(t_{n}-k\right)\right\|+\max _{1 \leqslant i \leqslant m} \mid e_{i}\left(t_{n}\right) / /\left\|\chi\left(t_{n}-k\right)\right\|
$$

Since $\left\|\chi\left(t_{n}-k\right)\right\| \rightarrow \infty$ as $t_{n} \rightarrow \infty$, the first term on the right-hand side of (33) tends towards zero. Equation (33) contradicts (31). Then the assumption that $\{\|\chi(t)\|\}$ is unbounded is false and the property (i) is proven. From Lemma 2 (ii) the property (ii) follows immediately.

From Theorem 1 one observes that the adaptive controller guarantees the BIBO stability of the closed loop system and makes the generalized errors $e_{i}(t)$ bounded. From (8) it is obvious that the unmodeled dynamics come from two parts, that is, plantmodel order mismatches and bounded disturbances. If the plant-model order mismatches, i.e. $r-r_{1}$ and $s-s_{1}$, are smaller and the norm of the unmodeled parameter vector, $\left\|\rho_{i}\right\|$, is also smaller, the upper bound $M_{i}$ may be decreased. In practical applications where the plant parameters are unknown, the values for $M_{i}, i=1, \ldots, m$, must be chosen reasonably according to the understanding of the plant.

\section{Conclusions}

Some improvements and extensions of earlier work (Cluett et al. 1987, 1988) have been made in this paper. The open-loop unstable and/or non-minimum phase plants can be handled by incorporating weight polynomial matrices into the controller. The combination of the normalization technique and the parameter estimation scheme with dead zones make the analysis of the robust stability and global convergence considerably simpler.

\section{REFERENCES}

Clarke, D. W. and Gawthrop, P. G. (1975). Self-tuning controller. IEE Proc. D., 122, 929-934. Clarke, D. W. and Gawthrop, P. G. (1979). Self-tuning control. IEE Proc. D., 126, 633-640. Cluett, W. R., Shah, S. L. and Fisher, D. G. (1987). Stable robust adaptive controller. Int. J. Control, 45, 1265-1273.

Cluett, W. R., Martin-Sanchez, J. M., Shah, S. L. and Fisher, D. G. (1988). Stable discretetime adaptive control in the presence of unmodeled dynamics. IEEE Trans. Automatic Control, 33, 410-414. 
96

Multivariable robust adaptive controller

Goodwin, G. C., Ramadge, P. J. and Canes, P. E. (1980). Discrete-time multivariable adaptive control. IEEE Trans. Automatic Control, 25, 449-456.

GU, X. Y. and WANG, W. (1988). Stable discrete time multivariable adaptive control in the presence of unmodeled dynamics. Propr. 8th IF AC Sump. on identification and system parameter estimation, pp. 265-270.

Korvo, H. N. (1980). A multivariable self-tuning controller. Automatica, 16, 351-366.

ORTEGA, R. and YU, T. (1987). Theoretical results on robustness of direct adaptive controller: a survey, Proc. IF AC World Congress, 14, pp. 1-15.

Rohrs, C. E., Valavani, L., Athens, M. and Stein, G. (1982). Robustness of adaptive control algorithms in the presence of unmodeled dynamics. Propr. Conf. Decis. Control, pp. 3-11. Wolovich, W. A. (1974). Linear multivariable systems. New York, Springer-Verlag, pp. 159. 